\title{
Zentralvorstandssitzung vom 19. Oktober 2011
}

\begin{abstract}
eHealth: Öffentliches Gesundheitsportal - Die FMH wird sich am Aufbau eines öffentlichen Gesundheitsportals beteiligen; als Trägerschaft kommen für den Zentralvorstand der Bund, die Kantone, zertifizierte NGO sowie themenbezogen auch Organisationen aus der Gesundheitswirtschaft in Frage.
\end{abstract}

Revision Medizinalberufegesetz - FMH und SIWF formulieren gemeinsam ihre Anliegen für die Revision des Medizinalberufegesetzes. Insbesondere möchten sie die nutzlose Verfügung beim Eintritt in die Weiterbildung abschaffen sowie den bürokratischen Aufwand in den Weiterbildungsprogrammen reduzieren.

Revision Transplantationsgesetz - Der Zentralvorstand verabschiedet die Stellungnahme zur Revision des Transplantationsgesetzes, die stark auf jener der Schweizerischen Akademie Medizinischer Wissenschaften basiert und von vielen Ärztegesellschaften unterstützt wird.
Telefonbucheinträge Chiropraktoren, Notfalldienst und Telemedizin - Der Zentralvorstand stimmt $\mathrm{zu}$,

- dass Chiropraktoren auf www.local.ch unter dem Stichwort «Ärzte» beim Buchstaben C unter «siehe Rubrik Chiropraktoren, ausserhalb Rubrik Ärzte» erwähnt werden sollen, sowie

- dass jeder Notfalldienst- und Telemedizinanbieter seinen Telefonbucheintrag vor dem Antrag auf local.ch bei der kantonalen Ärztegesellschaft bewilligen lassen muss.

Manifest Gesundheitspersonal - Schweizer Organisationen wollen den WHO-Kodex zur Rekrutierung von Gesundheitspersonal von 2010 mit einem Manifest bekräftigen. Die FMH unterstützt dieses Manifest, insbesondere seine Forderung nach mehr Medizin-Studienplätzen sowie höhere Investitionen in die Ausbildung von Ärztinnen und Ärzten.

\section{Findet Sie.} Das Ärzteverzeichnis doctorfmh.

\author{
Dort präsent sein, wo Patienten ihren Arzt \\ suchen. Kostenlos für Mitglieder.
}

